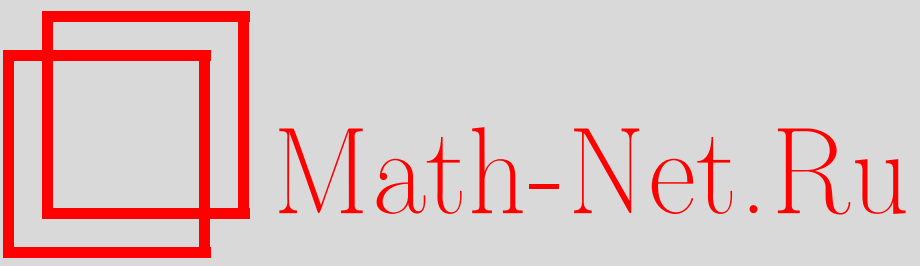

Ч. С. Ким, А. М. Сатанин, Дискретные уровни в континууме и туннелирование, TMФ, 1999, том 120, номер 1, 116-129

DOI: https://doi.org/10.4213/tmf764

Использование Общероссийского математического портала Math-Net.Ru подразумевает, что вы прочитали и согласны с пользовательским соглашением

http://www . mathnet.ru/rus/agreement

Параметры загрузки:

IP : 54.81 .137 .203

26 апреля 2023 г., $17: 30: 26$ 
ТЕОРЕТИЧЕСКАЯ

И МАТЕМАТИЧЕСКАЯ

ФИЗИКА

Том 120, № 1

июль, 1999

(C) 1999 г.

Ч. С. Ким* , А. М. Сатанин*

\section{ДИСКРЕТНЫЕ УРОВНИ В КОНТИНУУМЕ И ТУННЕЛИРОВАНИЕ}

Исследуется электронная локализация и туннелирование в квазиодномерном наноканале с двумя притягивающими примесями. Решена одноэлектронная задача для случая короткодействуюшего взаимодействия с примесью. Получены выражения для амплитуды рассеяния, волновой функции и электронного спектра. Показано, что когерентное взаимодействие резонансов Фано ведет к новому фазовокогерентному эффекту - коллапсу резонансно-антирезонансных пар и исчезновению резонансов. Как следствие в системе при вполне определенных критических параметрах появляются дискретные уровни в континууме. Представлен детальный анализ туннелирования электронов через дискретные уровни. Показано, что в этом случае может быть реализовано своеобразное вырождение, ког да одно состояние принадлежит дискретному спектру, а другое - распространяющемуся.

\section{1. ВВЕДЕНИЕ}

Согласно концепии, выдвинутой Моттом в теории локализации электронов [1], распространяющиеся электронные состояния (энергия которых принадлежит непрерывному спектру) и локализованные состояния (состояния дискретного спектра) отделены друг от друга определенной энергией - краем подвижности. Хорошо известно, что в статических транспортных явлениях принимают участие только распространяюшиеся электроны. Между тем основные принципы квантовой теории допускают существование дискретных уровней в континууме [2]. Примеры такого рода состояний известны в атомных системах $[3,4]$, однако их роль в квантовом транспорте не исследована. Интересно получить условия, при которых дискретные уровни могут появиться в континууме, и исследовать туннелирование электронов через дискретные уровни.

Квазиодномерные наноструктуры открывают новые возможности для проектирования квантовых состояний [5] и изучения нового типа квантовых когерентных эффектов. Кондактанс квантовой структуры $G$ (квантового волновода) может быть выражен че-

${ }^{*}$ Нижегородский государственный университет, Нижний Новгород, Россия. E-mail: satanin@phys.unn.runnet.ru 
рез прозрачность $T$ канала согласно известной формуле Ландауэра-Бюттикера $[6,7]$

$$
G=\frac{e^{2}}{h} T
$$

В свою очередь, прозрачность определяется электронной дифракцией на микроструктуре и интерференцией волн при рассеянии на примесях. Как это следует из решения уравнения Шредингера для электрона в квантовом канале без примесей, нижняя разрешенная энергия - энергия поперечного квантования - играет роль своеобразного края подвижности. Только электроны, имеюшие энергию выше края подвижности, могут распространятся в волноводе. В этой связи высшие зоны квантования также играют роль краев подвижности (для различных поперечных квантовых состояний). Если в канале присутствуют притягивающие примеси, то дискретные уровни могут появиться только ниже наинизшей зоны, тогда как ниже высших зон появляются только резонансные уровни. Это связано со взаимодействием дискретных уровней, отшепившихся от высших зон, с континуумом низколежащих зон. Проблема взаимодействия резонансов с континуумом представляет интерес также в связи с изучением квантования проводимости, поскольку примеси приводят к квантовой эрозии ступеней проводимости. В последнее время этой проблеме посвяшено большое число как экспериментальных $[8,9]$, так и теоретических [10-15] работ. В частности, в работах [11-15] было показано, что короткодействуюшие притягиваюшие примеси дают уединенные асимметричные резонансы Фано [16] в прозрачности. Ранее нами было показано, что в двухканальном приближении [17] взаимодействие резонансов Фано может приводить к коллапсу резонансов и появлению дискретных уровней в континууме.

В данной работе когерентное взаимодействие резонансов изучается в рамках модели короткодействуюших примесей в квантовом волноводе $[11,12]$. В случае симметричной конфигурации примесей найдено точное решение уравнения Шредингера для дискретных уровней в континууме. Показано, что волновая функция уровней может быть нормирована в том случае, если уровни лежат выше края подвижности первой зоны. Получено выражение для амплитуды рассеяния, позволяющее показать взаимодействие резонансов Брейта-Вигнера и резонансов Фано. Изучено поведение амплитуды рассеяния при критических параметрах системы, когда появляются дискретные уровни. Показано, что в канале могут существовать два типа состояний, принадлежаших разным классам - локализованным и распространяюшимся. Мы приводим аргументы, согласно которым эти два класса состояний в принципе могут быть созданы в канале путем выбора различных граничных условий.

В разделе 2 статьи решено уравнение Шредингера для электрона в канале с двумя примесями. Показана возможность появления дискретных уровней в континууме. В разделе 3 найдена матрица рассеяния и изучено взаимодействие резонансов Фано. Обсуждается структура амплитуды рассеяния для критических параметров системы, когда происходит туннелирование через дискретные уровни. В разделе 4 суммированы результаты и обсуждаются возможные следствия. 


\section{2. ЛОКАЛИЗОВАННЫЕ СОСТОЯНИЯ В КОНТИНУУМЕ}

Рассмотрим движение электрона в двумерном канале (волноводе) шириной $W$. Предполагается, что канал расположен в плоскости $(x, y)$ и электрон может распространяться вдоль оси $x$ между границами $y=0$ и $y=W$. Квантовые состояния в совершенном волноводе могут быть записаны в виде

$$
\psi_{n, k}^{(0)}(x, y)=e^{i k x} \varphi_{n}(y),
$$

где $k$ - волновой вектор электрона вдоль канала, $\varphi_{n}(y)$ - волновая функция в потенциале конфайнмента. Электронная энергия определяется выражением

$$
E_{n k}=\frac{\hbar^{2} k^{2}}{2 m}+E_{n}
$$

где $E_{n}$ - энергия поперечного движения (энергия квантования или край подвижности). Волновая функция в канале с примесями может быть записана в виде

$$
\Psi(x, y)=\sum_{n=1}^{\infty} \psi_{n}(x) \varphi_{n}(y)
$$

где $\psi_{n}(x)$ - функции, определяемые уравнением Шредингера

$$
-\frac{\hbar^{2}}{2 m} \frac{\partial^{2}}{\partial x^{2}} \psi_{n}(x)+\sum_{n^{\prime}=1}^{\infty} V_{n, n^{\prime}}(x) \psi_{n^{\prime}}(x)=\left(\mathcal{E}-E_{n}\right) \psi_{n}(x) .
$$

Будем полагать примеси короткодействующими. Это означает, что длина волны электрона велика по сравнению с масштабом примеси. Потенциал двух притягиваюших примесей можно записать в виде $[11,12]$

$$
V(x, y)=-\frac{\hbar^{2} \lambda}{m}\left[\delta\left(x-\frac{L}{2}\right)+\delta\left(x+\frac{L}{2}\right)\right] \delta\left(y-y_{s}\right),
$$

где безразмерный параметр $\lambda$ характеризует взаимодействие электрона с примесью; $\left( \pm L / 2, y_{s}\right)$ - координаты примеси. Здесь мы ограничимся рассмотрением только симметричной конфигурации, когда потенциал не меняется при замене $x \rightarrow-x$. В случае короткодействуюших примесей действие потенциала сводится к заданию граничных условий для волновых функций

$$
\begin{aligned}
\psi_{n}^{>} & =\psi_{n}^{<}, \\
\psi^{\prime}{ }_{n}-\psi^{\prime}{ }_{n} & =-2 \sum_{n^{\prime}=1}^{\infty} v_{n, n^{\prime}} \psi_{n^{\prime}}^{>},
\end{aligned}
$$

где $v_{n, n^{\prime}}=\lambda \varphi_{n} \varphi_{n^{\prime}}-$ матричные элементы примесного потенциала; $\varphi_{n} \equiv \varphi_{n}\left(y_{s}\right) ; \psi_{n}^{>}$, $\psi_{n}^{<}-$волновые функции в двух соседних (примыкаюших) областях. 
Покажем, что для энергий электрона, лежащих в интервале $E_{1}<\mathcal{E}<E_{2}$, уравнение Шредингера может иметь нормированное решение с дискретной энергией. Симметричное решение (1) (для четной функции $\psi_{n}(x)=\psi_{n}(-x)$ ) в области, где потенциал равен нулю, можно записать в виде

$$
\psi_{1}(x)= \begin{cases}a_{1} \cos \left(k_{1} x\right), & |x|<\frac{L}{2} \\ 0, & |x|>\frac{L}{2}\end{cases}
$$

а для всех $n \geqslant 2-$ как

$$
\psi_{n}(x)= \begin{cases}a_{n} \operatorname{ch}\left(\left|k_{n}\right| x\right), & |x|<\frac{L}{2}, \\ c_{n} e^{-\left|k_{n}\right||x|}, & |x|>\frac{L}{2}\end{cases}
$$

где $k_{n}=\sqrt{2 m\left(\mathcal{E}-E_{n}\right) / \hbar^{2}}, k_{n}=i\left|k_{n}\right|$ для $\mathcal{E}<E_{n}$.

Из граничных условий следует, что решения, определяемые формулами (2) и (3), справедливы, если

$$
\begin{aligned}
\cos \theta_{1} & =0 \\
\left|k_{n}\right| e^{\left|\theta_{n}\right|} a_{n} & =\sum_{n^{\prime}=2}^{\infty} v_{n, n^{\prime}} \operatorname{ch}\left(\left|\theta_{n^{\prime}}\right|\right) a_{n^{\prime}}
\end{aligned}
$$

где $\theta_{n}=k_{n} L / 2$ - фазы волн. Заметим, что при записи (2) и (3) неоднородные решения также были приняты во внимание; для $\mathcal{E}>E_{2}$ фазы решений записываются как $\theta_{n}=$ $i\left|\theta_{n}\right|$.

Для антисимметричного случая $\left(\psi_{n}(x)=-\psi_{n}(-x)\right)$ решения могут быть найдены по аналогии с симметричным случаем. Энергия определяется выражениями

$$
\begin{aligned}
\sin \theta_{1} & =0 \\
\left|k_{n}\right| e^{\left|\theta_{n}\right|} a_{n} & =\sum_{n^{\prime}=2}^{\infty} v_{n, n^{\prime}} \operatorname{sh}\left(\left|\theta_{n^{\prime}}\right|\right) a_{n^{\prime}}
\end{aligned}
$$

Отметим, что решения уравнений (5) и (7) были записаны в более обшем случае; они могут быть применимы для примесей, имеющих конечный размер в поперечном направлении. Однако решения (4)-(7) для произвольной матришы $v_{n, n^{\prime}}$ можно получить только численными методами. Более общее решение записано здесь и по причине, что при переходе к короткодействующей примеси необходима регуляризация выражений, которая может быть обоснована при обрашении к модели примесей с конечными размерами. Действительно, если примеси в канале имеют конечный размер в поперечном направлении $W_{a}$, то матричные элементы $v_{n, n^{\prime}}$ являются убывающими функциями $n$ и $n^{\prime}$. В этом случае суммы по $n^{\prime}$ в (5) и (7) хорошо определены. Например, если рассмотреть модель прямоугольной ямы, то характерное число слагаемых можно оценить как $n \sim W / W_{a}$. Для короткодействуюшей модели потенциала $v_{n, n^{\prime}}=\lambda \varphi_{n} \varphi_{n^{\prime}}$ и матричные элементы осциллируют в зависимости от $n$ и $n^{\prime}$. Физически очевидно, что когда 
длина волны электрона сравнима с величиной $W_{a}$, модель короткодействующей ямы не применима. Мы используем простейший метод регуляризации, обрезая суммы при $n \geqslant N=\left[W / W_{a}\right]$, где $[x]$ означает целую часть числа $x$.

Для короткодействуюшей модели перепишем формулу (5) в виде

$$
\begin{aligned}
& \left|k_{n}\right| e^{\left|\theta_{n}\right|} a_{n}=\lambda \varphi_{n} \nu, \\
& \nu=\sum_{n^{\prime}=2}^{N} \operatorname{ch}\left(\left|\theta_{n^{\prime}}\right|\right) a_{n}^{\prime},
\end{aligned}
$$

где вьполнено обрезание. Подставляя в (8) выражение для коэффициентов $a_{n}$, определяемое (9), получим вместо (5) уравнение

$$
1=2 \lambda \sum_{n=2}^{N} \frac{\operatorname{ch}\left(\left|\theta_{n}\right|\right)}{\left|k_{n}\right|} e^{-\left|\theta_{n}\right|} \varphi_{n}^{2} .
$$

Аналогично для антисимметричных решений (7) имеем

$$
1=2 \lambda \sum_{n=2}^{N} \frac{\operatorname{sh}\left(\left|\theta_{n}\right|\right)}{\left|k_{n}\right|} e^{-\left|\theta_{n}\right|} \varphi^{2}{ }_{n} .
$$

Таким образом, собственные значения спектральной проблемы суть решения уравнений $(4),(10)$ в симметричном случае и уравнений (6), (11) в антисимметричном.

Теперь перейдем к детальному обсуждению решений уравнений (4) и (10). Мы имеем здесь дело с двухпараметрической спектральной проблемой, поскольку два уравнения должны иметь решение одновременно. Будем использовать энергию $\mathcal{E}$ и расстояние меж ду примесями $L$ в качестве спектральных параметров. Решение уравнения (4) можно записать в виде

$$
E(j)=E_{1}+\frac{\hbar^{2} \pi^{2}(2 j+1)^{2}}{2 m L^{2}}, \quad j=0,1,2, \ldots .
$$

Подставляя формулу (12) в уравнение (10), получаем бесконечную систему уравнений, которая решается стандартными методами.

В качестве примера рассмотрим решение в двухканальном приближении. Для интервала энергии $E_{1}<\mathcal{E}<E_{2}$ двухканальное приближение дает качественно правильный ответ, поскольку в этом случае резонансно взаимодействуют состояния только двух каналов. Из уравнения (10) имеем

$$
\operatorname{th}\left(\left|\theta_{2}\right|\right)=\frac{u}{\left|\theta_{2}\right|}-1,
$$

где $u=\lambda \varphi_{1}{ }^{2} W$. Мы замечаем, что для произвольного расстояния $L$ уравнение (13) определяет уровни в двухъямной задаче [18], однако в данном случае эти уровни лежат 
ниже $E_{2}$. Поскольку уровни лежат в континууме, они имеют ширину вследствие взаимодействия с континуумом подзоны $n=1$. Однако ширина уровней может оказаться равной нулю, если уравнения (4) и (10) одновременно имеют решение. Следовательно, если параметры системы совпадают с критическими $(E(j), L(j))$, то в системе появляются дискретные уровни в континууме. Легко показать, что факт сушествования уровней не зависит от числа учитываемых каналов. В обшем случае бесконечная серия дискретных уровней располагается в интервале между двумя предельными энергиями $E_{0}$ и $E_{\infty}$, которые определяются из уравнения (10). Полагая в (10) параметр $L=0$, получаем уравнение

$$
1=2 \lambda \sum_{n=2}^{N} \frac{\varphi^{2}{ }_{n}}{\left|k_{n}\right|}
$$

для первой предельной энергии $E_{0}$. Вторая предельная энергия $E_{\infty}$ следует из (10), если положить $L \rightarrow \infty$,

$$
1=\lambda \sum_{n=2}^{N} \frac{\varphi^{2}}{\left|k_{n}\right|} .
$$

Решения уравнений для антисимметричного случая могут быть получены совершенно аналогично за исключением того, что в этом случае решение возможно только при $L>L_{0}$, где $L_{0}$ - минимальная длина, определяемая соотношением

$$
1=2 \lambda \sum_{n=2}^{N} \frac{\operatorname{sh}\left(\left|\theta_{n}\right|\right)}{\left|k_{n}\right|} e^{-\left|\theta_{n}\right|} \varphi_{n}^{2}
$$

(энергия $\mathcal{E}$ в формуле (14) должна быть положена равной энергии $E_{2}$ ). Другими словами, в квазиодномерном случае мы имеем условие на параметры, подобное тому, которое определяет условие существования уровня для антисимметричного состояния в двухъямной одномерной задаче [18].

Локализованным состояниям можно дать простую физическую интерпреташию. Они представляют собой стоячую волну с амплитудой $a_{1}$ между двумя “зеркалами” Фано и неоднородные волны в каналах с $n \geqslant 2$. Волновая функция в этих каналах убывает экспоненциально при удалении от примесей.

Нетрудно показать, что для энергий $\mathcal{E}>E_{2}$ уровни будут в обшем случае иметь ширину. Однако для специальных параметров примесей или канала ширина уровней может обратиться в нуль. Например, такие решения можно найти при $\varphi\left(y_{s}\right)=0$. В этом случае параметр $y_{s}$ может рассматриваться как дополнительный спектральный параметр.

Интересно отметить, что в случае, когда в качестве потенциала конфайнмента выбирается бесконечно глубокая яма, спектральные уравнения могут быть записаны как уравнения для двух безразмерных параметров $\epsilon=\mathcal{E} / E_{1}$ и $l=\pi L / 2 W$. Это означает, что все наши выводы будут справедливы, если вместо расстояния между примесями в качестве спектрального параметра можно использовать ширину канала $W$. 


\section{3. ТУННЕЛИРОВАНИЕ ЧЕРЕЗ ДИСКРЕТНЫЕ УРОВНИ В КОНТИНУУМЕ}

Получим выражение для амплитуды рассеяния электрона в канале с примесями. Решение уравнения Шредингера для падаюших и рассеянных волн записывается в виде

$$
\psi_{n}=A_{n} e^{i k_{n} x}+B_{n} e^{-i k_{n} x} \quad \text { для } x<-\frac{L}{2}
$$

$$
\psi_{n}=C_{n} e^{i k_{n} x} \quad \text { для } x>\frac{L}{2} .
$$

Решения в области $-L / 2<x<L / 2$ имеют вид

$$
\psi_{n}=a_{n} e^{i k_{n} x}+b_{n} e^{-i k_{n} x}
$$

При записи решений (15)-(17) учтены как распространяюшиеся, так и неоднородные решения с $k_{n}=i\left|k_{n}\right|$. Нужно только иметь в виду, что для тех каналов, для которых $\mathcal{E}<E_{n}$, амплитуды падающих волн следует положить равными нулю $\left(A_{n}=0\right.$ для всех $n$, когда $\left.\mathcal{E}<E_{n}\right)$ в силу условий при $x \rightarrow-\infty$. Подставляя выражения $(15)-(17)$ в граничные условия, после исключения промежуточных амплитуд $a_{n}$ и $b_{n}$ находим

$$
\begin{gathered}
C_{n}+\frac{e^{-i \theta_{n}}}{i k_{n}} \sum_{n^{\prime}=1}^{\infty} v_{n, n^{\prime}} e^{i \theta_{n^{\prime}}} C_{n^{\prime}}+\frac{e^{i \theta_{n}}}{i k_{n}} \sum_{n^{\prime}=1}^{\infty} v_{n, n^{\prime}} e^{i \theta_{n^{\prime}}} B_{n^{\prime}}= \\
=A_{n}-\frac{e^{i \theta_{n}}}{i k_{n}} \sum_{n^{\prime}=1}^{\infty} v_{n, n^{\prime}} A_{n^{\prime}}, \\
\frac{e^{i \theta_{n}}}{i k_{n}} \sum_{n^{\prime}=1}^{\infty} v_{n, n^{\prime}} e^{i \theta_{n^{\prime}}} C_{n^{\prime}}+B_{n}+\frac{e^{-i \theta_{n}}}{i k_{n}} \sum_{n^{\prime}=1}^{\infty} v_{n, n^{\prime}} e^{i \theta_{n^{\prime}} B_{n^{\prime}}}= \\
=\frac{e^{-i \theta_{n}}}{i k_{n}} \sum_{n^{\prime}=1}^{\infty} v_{n, n^{\prime}} e^{i \theta_{n^{\prime}} A_{n^{\prime}} .}
\end{gathered}
$$

Путем обрашения матриц слева в уравнениях (18) и (19) можно найти амплитуды $C_{n}$ и $B_{n}$, а следовательно, амплитуды прохождения и отражения, которые определяются соотношениями

$$
C_{n}=\sum_{n^{\prime}} t_{n, n^{\prime}} A_{n^{\prime}}, \quad B_{n}=\sum_{n^{\prime}} r_{n, n^{\prime}} A_{n^{\prime}} .
$$

Для короткодействуюшей модели проблема обрашения матриш упрощается. В этом случае имеем

$$
\begin{aligned}
& C_{n}+\lambda \frac{e^{-i \theta_{n}}}{i k_{n}} \varphi_{n} \delta+\lambda \frac{e^{i \theta_{n}}}{i k_{n}} \varphi_{n} \beta=A_{n}-\frac{e^{i \theta_{n}}}{i k_{n}} \varphi_{n} \alpha, \\
& \lambda \frac{e^{i \theta_{n}}}{i k_{n}} \varphi_{n} \delta+B_{n}+\lambda \frac{e^{-i \theta_{n}}}{i k_{n}} \varphi_{n} \beta=-\frac{e^{-i \theta_{n}}}{i k_{n}} \varphi_{n} \alpha
\end{aligned}
$$


где

$$
\alpha=\sum_{n=1}^{N} e^{-i \theta_{n}} \varphi_{n} A_{n}, \quad \beta=\sum_{n=1}^{N} e^{i \theta_{n}} \varphi_{n} B_{n}, \quad \delta=\sum_{n=1}^{N} e^{i \theta_{n}} \varphi_{n} C_{n} .
$$

Умножая уравнение (21) на $e^{i \theta_{n}} \varphi_{n}$ и суммируя по $n$, получаем уравнение для $\beta$ в форме

$$
\beta=-\lambda \frac{\alpha S_{0}+\delta S_{1}}{1+\lambda S_{0}}
$$

где

$$
S_{0}=\sum_{n=1}^{N} \frac{\varphi_{n}^{2}}{i k_{n}}, \quad S_{1}=\sum_{n=1}^{N} \frac{e^{2 i \theta_{n}}}{i k_{n}} \varphi_{n}^{2} .
$$

Подставляя (22) в (20), получаем бесконечную систему уравнений

$$
C_{n}+\lambda \frac{e^{-i \theta_{n}}}{i k_{n}} \varphi_{n} \frac{1+\lambda\left(S_{0}-e^{2 i \theta_{n}} S_{1}\right)}{1+\lambda S_{0}} \delta=A_{n}-\frac{e^{i \theta_{n}}}{i k_{n}} \varphi_{n} \frac{\alpha}{1+\lambda S_{0}},
$$

которая связывает векторы $C_{n}$ и $A_{n}$. Матрица в левой части соотношения (23) имеет вид

$$
M_{n, n^{\prime}}=\delta_{n, n^{\prime}}+p_{n} q_{n^{\prime}} \varphi_{n} \varphi_{n^{\prime}}
$$

где

$$
p_{n}=\frac{e^{-i \theta_{n}}}{i k_{n}} \frac{1+\lambda\left(S_{0}-e^{2 i \theta_{n}} S_{1}\right)}{1+\lambda S_{0}}, \quad q_{n^{\prime}}=e^{-i \theta_{n^{\prime}}}
$$

Обратная матрица

$$
M_{n, n^{\prime}}^{-1}=\delta_{n, n^{\prime}}-\frac{p_{n} q_{n^{\prime}}}{Q} \varphi_{n} \varphi_{n^{\prime}},
$$

где

$$
Q=1+\sum_{n=1}^{N} p_{n} q_{n} \varphi_{n}^{2} .
$$

Применяя (24) для обрашения матрицы (23), находим после простых вычислений связь между амплитудами рассеянных $C_{n}$ и падаюших $A_{n}$ волн. Связь между $B_{n}$ и $A_{n}$ может быть найдена аналогичным методом. Таким образом, матрицы прохождения и отражения определяются выражениями

$$
\begin{aligned}
t_{n, n^{\prime}} & =\delta_{n, n^{\prime}}-2 \lambda \frac{\varphi_{n} \varphi_{n^{\prime}}}{i k_{n} D_{s} D_{a}}\left(\sin \theta_{n} \sin \theta_{n^{\prime}} D_{s}+\cos \theta_{n} \cos \theta_{n^{\prime}} D_{a}\right), \\
r_{n, n^{\prime}} & =-2 \lambda \frac{\varphi_{n} \varphi_{n^{\prime}}}{i k_{n} D_{s} D_{a}}\left(-\sin \theta_{n} \sin \theta_{n^{\prime}} D_{s}+\cos \theta_{n} \cos \theta_{n^{\prime}} D_{a}\right),
\end{aligned}
$$

где полюсные функции определены равенствами

$$
\begin{aligned}
& D_{s}=1+2 \lambda \sum_{n=1}^{N} \frac{\cos \theta_{n}}{i k_{n}} e^{i \theta_{n}} \varphi_{n}^{2}, \\
& D_{a}=1-2 \lambda \sum_{n=1}^{N} \frac{\sin \theta_{n}}{k_{n}} e^{i \theta_{n}} \varphi_{n}^{2} .
\end{aligned}
$$




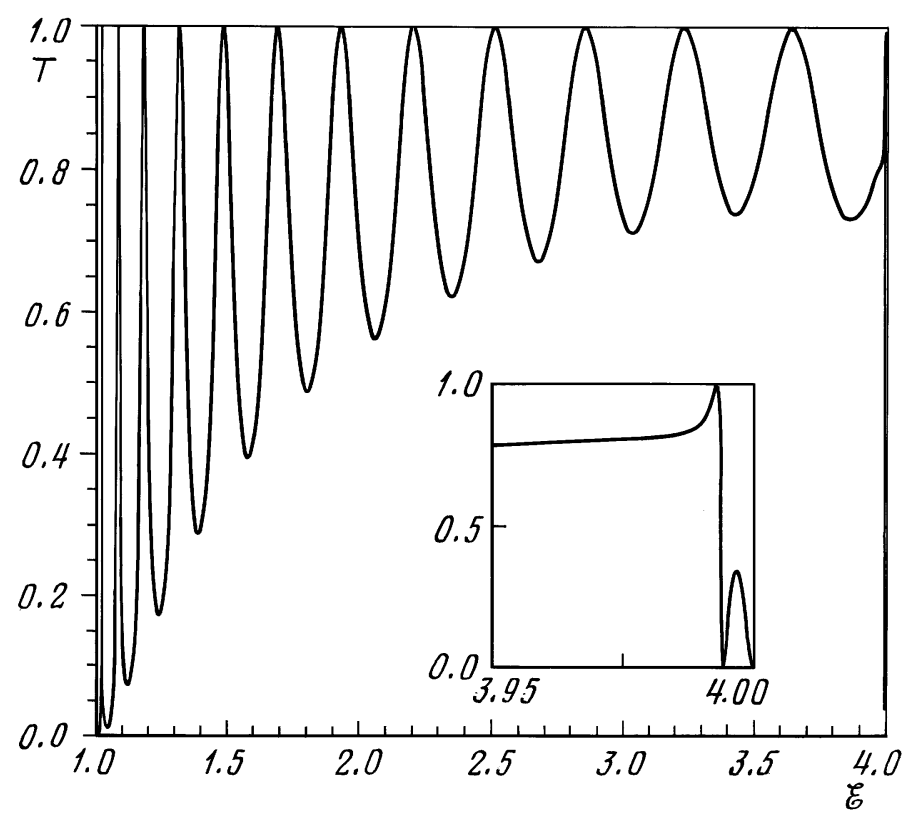

Рис. 1

Важно отметить, что суммирование по промежуточным каналам в равенствах (27), (28) учитывает как распространяюшиеся, так и неоднородные решения.

Можно проверить, что если расстояние $L \rightarrow 0$, то уравнения $(25)$ и $(26)$ дают

$$
t_{n, n^{\prime}}=\delta_{n, n^{\prime}}-2 \lambda \frac{\varphi_{n} \varphi_{n^{\prime}}}{i k_{n} D}, \quad r_{n, n^{\prime}}=-2 \lambda \frac{\varphi_{n} \varphi_{n^{\prime}}}{i k_{n} D}
$$

где

$$
D=1+2 \lambda \sum_{n=1}^{N} \frac{\varphi_{n}^{2}}{i k_{n}}
$$

Этот результат был ранее получен в работе [12] путем решения уравнений Дайсона.

Исследуем нули и полюсы амплитуды прохождения (25) для энергии частицы в интервале $E_{1}<\mathcal{E}<E_{2}$. Для амплитуды рассеяния между открытыми каналами $1 \rightarrow 1$ получаем

$$
t_{11}=\frac{D_{s} D_{a}-2 \lambda \frac{\varphi_{1}^{2}}{i k_{1}}\left(\sin ^{2} \theta_{1} D_{s}+\cos ^{2} \theta_{1} D_{a}\right)}{D_{s} D_{a}} .
$$

Из выражения (29) следует, что имеются два типа полюсов, которые определяются соотношениями

$$
D_{s}=2 \lambda \frac{\cos \theta_{1}}{i k_{1}} e^{i \theta_{1}} \varphi_{1}^{2}+1-2 \lambda \sum_{n=2}^{N} \frac{\operatorname{ch}\left(\left|\theta_{n}\right|\right)}{\left|k_{n}\right|} e^{-\left|\theta_{n}\right|} \varphi_{n}^{2}=0
$$


или

$$
D_{a}=-2 \lambda \frac{\sin \theta_{1}}{k_{1}} e^{i \theta_{1}} \varphi_{1}^{2}+1-2 \lambda \sum_{n=2}^{N} \frac{\operatorname{sh}\left(\left|\theta_{n}\right|\right)}{\left|k_{n}\right|} e^{-\left|\theta_{n}\right|} \varphi_{n}^{2}=0 .
$$

Ясно, что эти два типа полюсов связаны соответственно с симметричными и антисимметричными виртуальными состояниями в промежуточных каналах при рассеянии на примесях. Выражения для нулей амплитуды определяются из уравнения

$$
D_{s} D_{a}-2 \lambda \frac{\varphi_{1}^{2}}{i k_{1}}\left(\sin ^{2} \theta_{1} D_{s}+\cos ^{2} \theta_{1} D_{a}\right)=0
$$

Из уравнений (30)-(32) видно, что в общем случае они имеют комплексные решения. Можно различить два типа резонансов: резонансы Брейта-Вигнера и резонансы Фано. Первые связаны с интерференцией волн между примесями, а вторые - со взаимодействием дискретных уровней с континуумом. Два типа резонансов видны на рис. 1, где изображена прозрачность системы $T=\left|t_{11}(E)\right|^{2}$ в зависимости от энергии $\mathcal{E}$ для некоторого типичного расстояния между примесями $L=7.64 \mathrm{~W}$, а параметр связи всюду далее полагается $\lambda=0.115$.

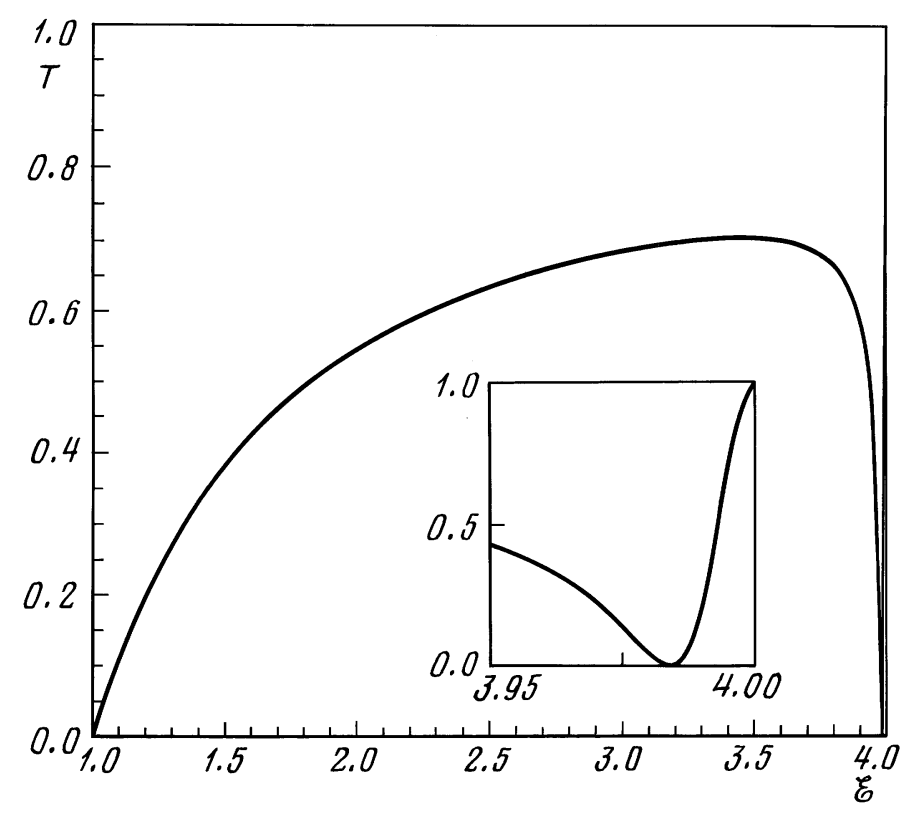

Рис. 2

Для выбранных параметров резонансы Фано лежат ниже дна второй подзоны размерного квантования - ниже второго края подвижности. Путем выбора специальных значений параметров можно сдвинуть резонансы Брейта-Вигнера далеко в комплексную плоскость так, чтобы остались только резонансы Фано. Мы изобразили прозрачность для такой ситуации на рис. 2, выбрав расстояние между примесями меньше, чем 


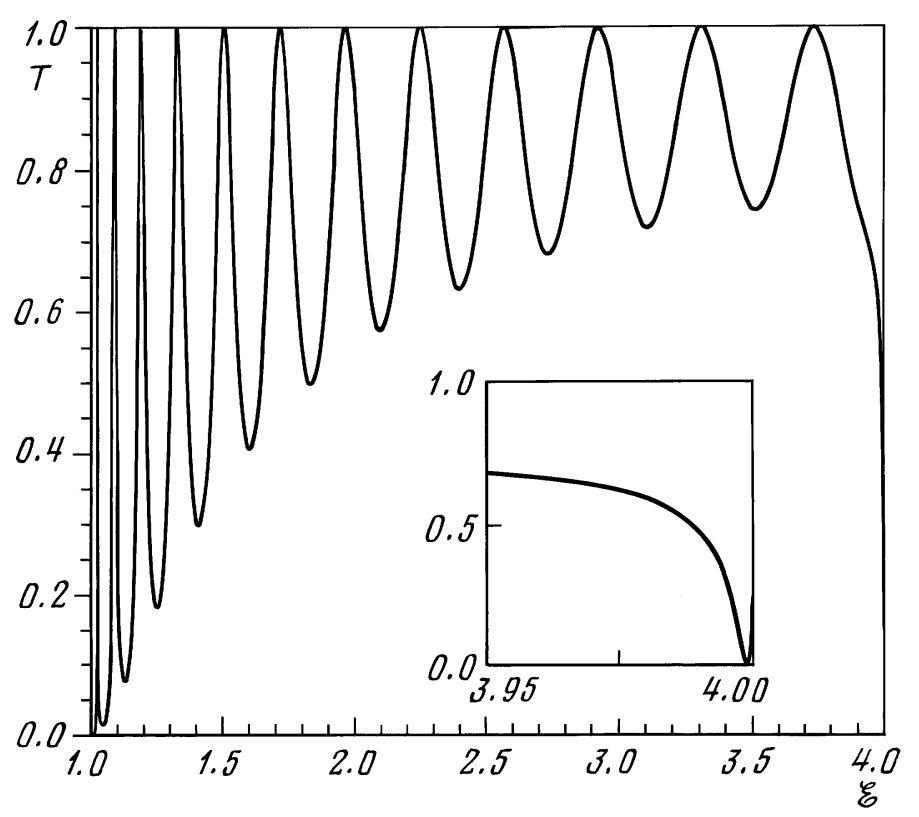

Рис. 3

длина волны частицы. В этом случае виден уединенный резонанс Фано, обусловленный квазисвязанным симметричным уровнем в двухъямной задаче.

В случае обшего положения примесей в канале наблюдается весьма сложное взаимодействие резонансов различных типов. Для упрощения анализа далее мы сосредоточим внимание на случае слабой связи. Пусть безразмерный параметр связи $\lambda$ мал. Тогда мы можем найти приближенное комплексное решение $D_{s}=0$ уравнения в виде $\mathcal{E}=E_{p}-i \Gamma$. В рамках пертурбативного подхода при решении уравнения (32) нули амплитуды $E_{0}$ близки к полюсам в комплексной плоскости. При этом вблизи полюса и нуля амплитуда характеризуется тремя параметрами $\left(E_{0}, E_{p}, \Gamma\right)$ и имеет форму асимметричного резонанса Фано [16]

$$
t_{11}(\mathcal{E}) \sim \frac{\mathcal{E}-E_{0}}{\mathcal{E}-E_{p}+i \Gamma} .
$$

В соответствии с нашим рассмотрением в разделе 2 дискретные уровни появляются для тех значений $L$ и $E$, для которых имеют место решения уравнений (4) и (10) (или (6) и (11)).

Пусть теперь расстояние равно критическому $L(j)$. Изучим зависимость амплитуды от энергии вблизи критического значения $E(j)$. Для $\mathcal{E}=E(j)$ уравнение $D_{s}=0$ может быть записано в точности, как два уравнения (4) и (10). В этом случае полюс выходит на реальную ось энергии. Из уравнения для нуля (32) мы видим, что оно также имеет место для той же самой энергии $E(j)$. Это означает, что для критических значений параметров имеем $\Gamma=0, E_{p}=E_{0}=E(j)$ и амплитуда прохождения электрона через 

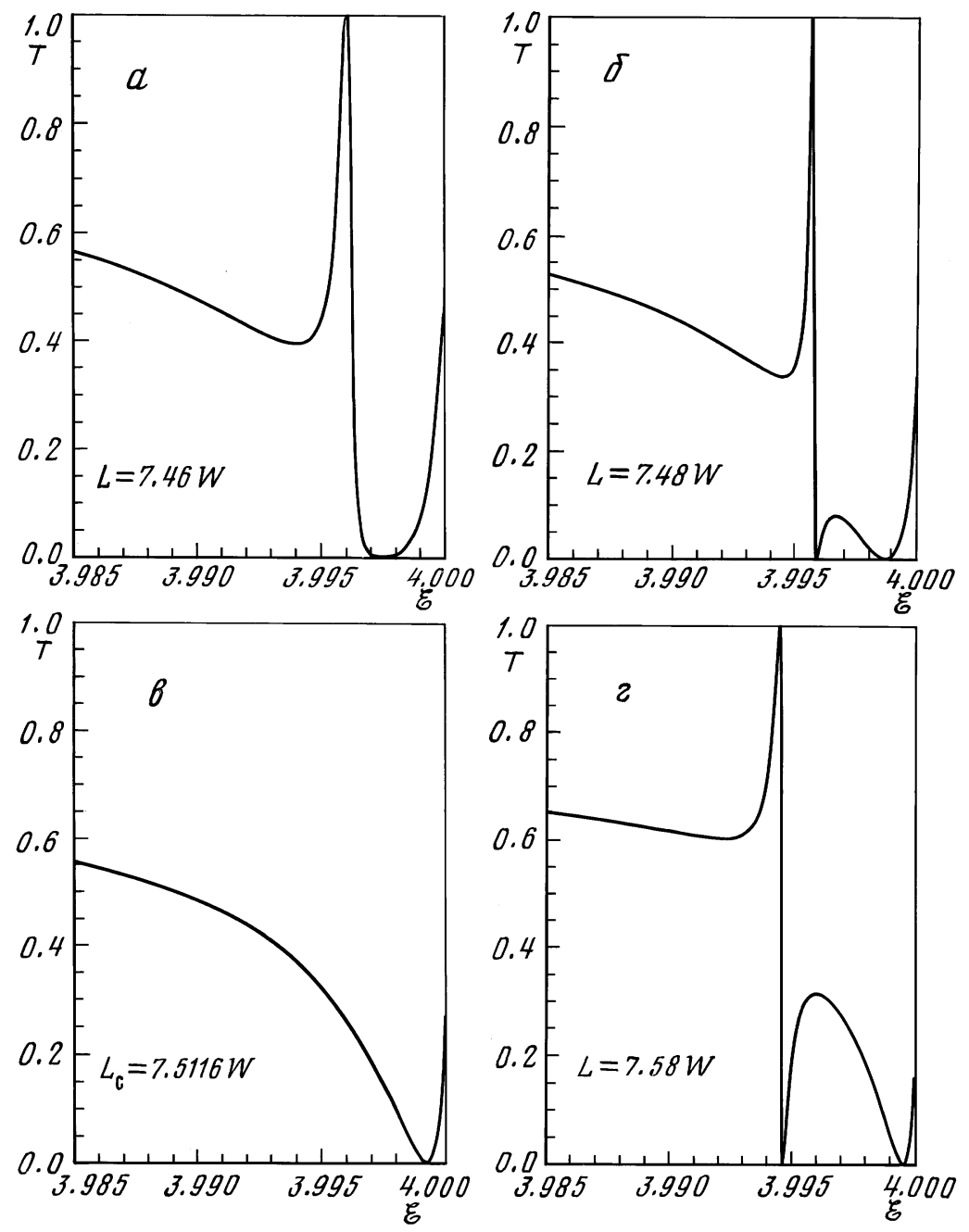

Рис. 4

дискретный уровень конечна. При этом резонанс Фано исчезает в прозрачности (см. рис. 3 , где расстояние между примесями $L=0.06 \mathrm{~W})$.

Для более детального анализа эффекта исчезновения резонансов на рис. 4 представлена прозрачность как функция энергии для нескольких расстояний $L=7.46 \mathrm{~W}, 7.48 \mathrm{~W}$, $7.5116 \mathrm{~W}, 7.58 \mathrm{~W}$ вблизи критического значения $L_{\mathrm{c}}=L(6)=7.5116 \mathrm{~W}$. Критическое расстояние было рассчитано путем численного решения уравнений (4) и (10). Результат показывает, что когда $L$ достигает критического значения $L_{\mathrm{c}}$, резонанс Фано исчезает. При этом прозрачность конечна для критического значения энергии $E_{\mathrm{c}}=E(6)=$ $3.99517 E_{1}$. Из рис. 4г видно, что резонансы появляются снова, когда расстояние проходит через критическое. 
Таким образом, показано, что в квазиодномерном канале возможны два типа состояний при одной и той же энергии - локализованное и распространяющееся. Эти два состояния в принципе могут быть созданы путем выбора двух различных типов граничных и начальных условий. Например, если волновые пакеты, имеюшие узкое распределение по энергии вблизи критического значения энергии, генерируются в промежутке меж ду примесями, то можно создать локализованные состояния. Распространяющиеся состояния могут быть реализованы путем создания пакетов, набегающих из внешней области.

\section{4. ОБСУЖДЕНИЕ РЕЗУЛЬТАТОВ}

В данной работе мы изучили рассеяние электрона на короткодействующих примесях в квазиодномерном квантовом канале. Данная проблема качественно отличается от аналогичной одномерной или двумерной проблемы, поскольку спектр электронов в канале квазиодномерный и имеется бесконечная серия краев подвижности. В этом случае дискретные примесные уровни могут попадать в континуум низколежаших зон. Показано, что притягиваюшие примеси вызывают появление асимметричных резонансов Фано в прозрачности. Когерентное взаимодействие резонансов приводит к появлению дискретных уровней в непрерывном спектре и качественной перестройке амплитуды рассеяния. Мы ввели двухпараметрическую спектральную проблему, которая определяет критические параметры системы, когда происходит коллапс резонансов. Волновая функция дискретных уровней в континууме найдена в явной форме.

Было изучено также туннелирование электрона через дискретные уровни и показано, что электрон может проходить через канал, когда его энергия совпадает с энергией дискретного уровня. При этом возможно существование двух типов состояний в канале, когда одно принадлежит дискретному спектру, а другое - распространяюшемуся. Такое своеобразное вырождение объясняется тем, что можно создать два типа состояний путем выбора различных граничных условий.

Представим оценку критических параметров системы, чтобы показать, что эти параметры могут быть реализованы методами современной нанотехнологии. Рассмотрим типичный $2 \mathrm{D}$-канал шириной $W$. Для такой структуры наинизшая энергия размерного квантования определяется как $E_{1}=\pi^{2} \hbar^{2} /\left(2 m W^{2}\right)$. Используя приведенное выше значение для параметра $\lambda$, находим минимальное критическое расстояние $L_{\mathrm{c}}=L(0)=$ $0.578 W$ и минимальную критическую энергию $E_{\mathrm{c}}=E(0)=3.989 E_{1}$. Искусственные примеси с заданными параметрами могут быть созданы в канале так, как это описано, например, в статье [19].

Благодарности. Работа поддержана KOSEF и Министерством образования Кореи (гранты № BSRI-96-2431 и BSRI-97-2431). Один из нас (А.М.С.) признателен KOSEF и Российскому фонду фундаментальных исследований (грант № 97-02-16923a) за поддержку. 


\section{Список литературы}

[1] N. F. Mott, E. A. Devis. Electronic Processes In Non-Crystalline Materials. Oxford: Claremont Press, 1979.

[2] J. von Neumann, E. Wigner. Z. Phys. 1929. V. 30. P. 465.

[3] F. H. Stillinger, D. R. Herrick. Phys. Rev. A. 1975. V. 11. P. 446.

[4] H. Friedrich, D. Wintgen. Phys. Rev. A. 1985. V. 31. P. 3964.

[5] Quantum Transport in Ultrasmall Devices. Proc. of a NATO Advanced Study Inst. Quantum Transport in Ultrasmall Devices, Il Ciocco, 1994. V. B 342. Eds. D.K. Ferry, H. L. Grubin, C. Jacoboni, A.-P. Jauho. New York: Plenum, 1995.

[6] R. Landauer. Philos. Mag. 1970. V. 21. P. 863.

[7] M. Buttiker. Phys. Rev. B. 1987. V. 35. P. 4123.

[8] P. L. McEuen et all. Surf. Sci. 1990. V. 229. P. 312.

[9] C.-T. Liang et all. Phys. Rev. B. 1997. V. 55. P. 6723.

[10] C. S. Chu, R. S. Sorbello. Phys. Rev. B. 1989. V. 40. P. 5941.

[11] P.F. Bagwell. Phys. Rev. B. 1990. V. 41. P. 10354.

[12] P. F. Bagwell. J. Phys.: Cond. Matter. 1990. V. 2. P. 6179.

[13] E. Tekman, S. Ciraci. Phys. Rev. B. 1990. V. 42. P. 9098.

[14] E. Tekman, P. F. Bagwell. Phys. Rev. B. 1993. V. 48. P. 2553.

[15] J. U. Nöckel, A. D. Stone. Phys. Rev. B. 1994. V. 50. P. 17415.

[16] U. Fano. Phys. Rev. 1961. V. 124. P. 1866.

[17] Ч. С. Ким, А. М. Сатанин. ЖЭТФ. 1999. Т. 115. С. 211.

[18] Л. Д. Ландау, Е. М. Лифииц. Квантовая механика. М.: Наука, 1989.

[19] S. Yamada, M. Yamamoto. Appl. Phys. Lett. 1996. V. 79. P. 8391.

Поступила в редакцию 24.XI.1998 г., после доработки 19.II.1999 г. 